VOLUME 33, ARTICLE 14, PAGES 391-424 PUBLISHED 1 SEPETEMBER 2015

http://www.demographic-research.org/Volumes/Vol33/14/

DOI: $\quad$ 10.4054/DemRes.2015.33.14

Research Article

Decomposing changes in life expectancy:

Compression versus shifting mortality

\author{
Marie-Pier Bergeron-Boucher
}

Marcus Ebeling

Vladimir Canudas-Romo

(C) 2015 Bergeron-Boucher, Ebeling \& Canudas-Romo.

This open-access work is published under the terms of the Creative Commons Attribution NonCommercial License 2.0 Germany, which permits use, reproduction \& distribution in any medium for non-commercial purposes, provided the original author(s) and source are given credit. See http://creativecommons.org/licenses/by-nc/2.0/de/ 


\section{Table of Contents}

$1 \quad$ Background $\quad 392$

$2 \quad$ Methods and data $\quad 394$

$2.1 \quad$ Decomposing life expectancy 394

2.2 Decomposing senescent mortality: Gompertz 396

2.3 Extending the model beyond senescent mortality 398

$\begin{array}{ll}\text { 2.3.1 } & \text { Gompertz-Makeham } \\ 2.3 .29\end{array}$

$\begin{array}{lll}2.3 .2 & \text { Siler } & 399\end{array}$

$\begin{array}{lll}2.4 & \text { Data } & 401\end{array}$

$3 \quad$ Illustration $\quad 402$

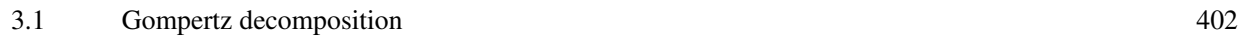

$\begin{array}{ll}3.2 & \text { Gompertz-Makeham decomposition }\end{array}$

$\begin{array}{lll}3.3 & \text { Siler decomposition } & 405\end{array}$

$\begin{array}{ll}3.4 & \text { Life expectancy and modal age at death }\end{array}$

$4 \quad$ Discussion and conclusion $\quad 409$

5 Acknowledgements $\quad 410$

$\begin{array}{lll}\text { A } & \text { Appendices } & 415\end{array}$ 


\title{
Decomposing changes in life expectancy: Compression versus shifting mortality
}

\author{
Marie-Pier Bergeron-Boucher ${ }^{1}$ \\ Marcus Ebeling ${ }^{2}$ \\ Vladimir Canudas-Romo ${ }^{1}$
}

\section{Abstract}

\section{BACKGROUND}

In most developed countries, mortality reductions in the first half of the 20th century were highly associated with changes in lifespan disparities. In the second half of the 20th century, changes in mortality are best described by a shift in the mortality schedule, with lifespan variability remaining nearly constant. These successive mortality dynamics are known as compression and shifting mortality, respectively.

\section{OBJECTIVE}

To understand the effect of compression and shifting dynamics on mortality changes, we quantify the gains in life expectancy due to changes in lifespan variability and changes in the mortality schedule, respectively.

\section{METHODS}

We introduce a decomposition method using newly developed parametric expressions of the force of mortality that include the modal age at death as one of their parameters. Our approach allows us to differentiate between the two underlying processes in mortality and their dynamics.

\section{RESULTS}

An application of our methodology to the mortality of Swedish females shows that, since the mid-1960s, shifts in the mortality schedule were responsible for more than $70 \%$ of the increase in life expectancy.

\section{CONCLUSIONS}

The decomposition method allows differentiation between both underlying mortality processes and their respective impact on life expectancy, and also determines when and how one process has replaced the other.

\footnotetext{
${ }^{1}$ Max-Planck Odense Center on the Biodemography of Aging, University of Southern Denmark, Odense, Denmark.

${ }^{2}$ Max Planck Institute for Demographic Research, Rostock, Germany. University of Rostock, Institute of Sociology and Demography, Rostock, Germany.
} 


\section{Background}

Human mortality has undergone remarkable declines over the years. The increase in life expectancy is probably the best expression for the dramatic mortality decline in the last 170 years (Oeppen and Vaupel 2002). Improvements in living conditions, nutrition and medicine are among the main reasons for this development (Riley 2001; Oeppen and Vaupel 2002). These changes in economic, social, and sanitary conditions first triggered an important decline in infant, child, and early adult mortality, which contributed to the reduction in lifespan disparities (Wilmoth and Horiuchi 1999; Edwards and Tuljapurkar 2005; Vaupel, Zhang, and van Raalte 2011). As individuals became more homogeneous in their ages at death, a compression of the distribution of deaths in a more narrow ageinterval was observed in many low-mortality countries in the first half of the twentieth century (Fries 1980; Wilmoth and Horiuchi 1999; Kannisto 2000, 2001; Cheung et al. 2009). Fries (1980) hypothesized that this dynamic can be interpreted as a compression of deaths against the upper limit of the human lifespan. Assuming a nearly negligible role for premature mortality, he stated the limit of the average age at death as approximately 85 years, with $95 \%$ of all deaths occurring in an age range of 4 years deviation (Fries 1980). The "compression of mortality hypothesis" motivated a rich discussion on the occurrence and interpretation of this development. Several studies provided evidence for a compression, but emphasized that the achieved mortality levels differ substantially from Fries' predictions (Nusselder and Mackenbach 1996; Wilmoth and Horiuchi 1999; Cheung et al. 2005).

After the period of strong compression, low-mortality countries entered a new era of change. Since the second half of the twentieth century, the main contributions to the increase in average age at death shifted from infant and early adult ages to old and very old-ages (Christensen et al. 2009). This generated changes in the mechanisms behind the increase in life expectancy (Wilmoth and Horiuchi 1999; Edwards and Tuljapurkar 2005; Smits and Monden 2009). The new mechanism behind improvement in life expectancy is best illustrated by a shift in the distribution of death toward older ages with a shape remaining nearly constant (Yashin et al. 2001; Bongaarts 2005; Cheung et al. 2005; Cheung and Robine 2007; Canudas-Romo 2008). Vaupel (1986), Vaupel and Gowan (1986) and Bongaarts (2005) were among the first to articulate the idea of shifting mortality. Canudas-Romo (2008) deepens this idea by studying the variability around and the change of the modal age at death. He finds that over time mortality shifts to higher ages, with approximately constant variability in age at death. He concludes that the shifting mortality pattern might be the new dynamic behind mortality improvements, subsequent to the compression process.

The ages at which mortality reductions occur tend to determine the dominating mortality dynamic: compression or shift. Compression is more pronounced when mortality reductions occur at very young and adult ages (Nusselder and Mackenbach 1996; 
Wilmoth and Horiuchi 1999; Kannisto 2000; Cheung et al. 2005). On the other hand, shifting mortality requires changes at old and very old-ages (Canudas-Romo 2008). Vaupel, Zhang, and van Raalte (2011) report relatively stable variability patterns for survivors beyond age 50 in the last 100 years. Engelman, Caswell, and Agree (2014) and Engelman, Canudas-Romo, and Agree (2010), however, provide evidence for a modest expansion of lifespan variability for survivors at older ages, resulting from mortality improvement at these same ages.

The measurement of compression and shifting mortality is an important issue, as both dynamics translate differently into survival, mortality density and hazard distributions (Wilmoth and Horiuchi 1999). Alterations are, however, visible in all three functions due to their interrelation. For instance, in a mortality compression context, the survival curve becomes more rectangular with increasing concentration of deaths at old-age, which is a well-known phenomenon called rectangularization (Nusselder and Mackenbach 1996; Wilmoth and Horiuchi 1999; Cheung et al. 2005). Simultaneously, the old-age bulk of deaths in the distribution of death becomes more pronounced, thereby reducing variability of the age at death. In the hazard distribution, the slope becomes steeper, with mortality reductions being more pronounced at younger ages (Wilmoth and Horiuchi 1999; Robine 2001).

In a shifting mortality context, these three functions also undergo transformations. The downward slope of the survival curve will shift to higher ages with an equal shape. Similarly, the density distribution will shift towards older ages with a shape also remaining constant. In the hazard distribution, the same pattern requires a constant slope depicted by a parallel shift of the logarithmic force of mortality toward higher ages (Bongaarts 2005; Canudas-Romo 2008). In this context, Bongaarts (2005) suggested fixing the shape parameter of mortality models and assumed that only scale and background parameters can vary over time. Vaupel (2010) also describes a postponement of senescence rather than a fundamental change of the age-pattern of mortality for the period starting around 1950 .

In the assessment of the shifting mortality period, the modal age at death has been an extensively used indicator. By shifting the modal age at death towards older ages, the deaths around this age move along with it (Canudas-Romo 2008). This indicator also has several advantages in the investigation of survival at old-ages. First, it is nonsensitive to mortality changes at younger ages. Second, it reflects the most common lifespan. Third, a change of the modal age can only be realized if there are pulling forces, meaning mortality improvement at ages older than the mode (Kannisto 2000; Canudas-Romo 2010). In fact, the modal age at death has shown an accelerated pace of increase since the onset of the old-age mortality decline (Kannisto 2000; Wilmoth and Robine 2003; Canudas-Romo 2008). Since the beginning of the 21 st century, this indicator has received increasing attention and has become a key indicator of lifespan, especially since longevity extension became determined by adult and old-age mortality (Kannisto 2000, 
2001; Bongaarts 2005; Cheung and Robine 2007; Canudas-Romo 2008, 2010; Ouellette and Bourbeau 2011; Horiuchi et al. 2013).

Therefore, compression and shifting mortality are observed respectively by changes in the variability of the age at death and in the modal age at death. Both dynamics also have different implications regarding changes in mortality: the former reflects changes in lifespan disparities, while the latter provides information about changes in the timing of mortality.

Considering the two periods of change in mortality development, two questions arise. First, what is the impact of compression and shifting mortality dynamics on the increase of life expectancy over time? Second, how and to what extent did one process replace the other? Additionally, considering the impact of child and young adult mortality reductions on the appearance of compression, one might further ask, if only adult and old-ages mortality is analyzed, how does the impact of both dimensions change?

To approach these questions, a new methodology to study changes in compression and shifting mortality over time and their effect on life expectancy is presented. We quantify the gains in life expectancy due to changes in the timing of mortality and changes in lifespan disparities, respectively. Using newly developed parametric expressions of the force

of mortality (Horiuchi et al. 2013; Missov et al. 2015), we decompose the change in life expectancy between two distributions by the contribution of a shift in the modal age at death and a change in variability of the age at death.

This paper is divided into four sections, with this background as the first section. In the following section, we introduce the decomposition methodology, at first in general terms and then for the Gompertz, Gompertz-Makeham and Siler models. The third section presents an illustration of the methodology applied to discrete data, followed by the fourth section, in which we present our conclusions.

\section{Methods and data}

\subsection{Decomposing life expectancy}

In order to explain the dynamics behind changes in mortality, demographers have developed several techniques to decompose changes in life expectancy by different components of mortality, such as ages and causes of death. Some methods focus on discrete differences between two life expectancies (Pollard 1982; Arriaga 1984; Pressat 1985; Andreev, Shkolnikov, and Begun 2002; Firebaugh et al. 2014) while others consider continuous changes (Vaupel 1986; Keyfitz 1977; Vaupel and Canudas-Romo 2003; Beltrán-Sánchez, Preston, and Canudas-Romo 2008; Horiuchi, Wilmoth, and Pletcher 2008). We follow the latter approach of a continuous decomposition of changes in life expectancy by vari- 
ability and shifting effects using a recent expression of the Gompertz mortality model.

Figure 1 shows the distribution of deaths for Gompertz parameters under two scenarios. It illustrates how changes in mortality can be decomposed into effects due to changes in variability and the shifting of mortality. Assuming a general change of mortality between the two distributions (in Figure 1 as the arrow denoted as A), the shifting effect is the hypothetical change resulting only if the modal age at death $(M)$ would have changed between those two distributions (in Figure 1 as arrow B). The variability effect is the hypothetical change produced only if the slope of the hazard function $(\beta)$ changes from one distribution to another (in Figure 1 as arrow $\mathrm{C}$ ). The latter transformation $\mathrm{C}$, of changing the slope of the hazard distribution, also changes the shape of the density distribution, and thus their variability (Wilmoth 1997).

Changes in life expectancy at birth over time (denoted as $\dot{e}_{0, t}$ ) can thus be decomposed into two components

$$
\dot{e}_{0, t}=\Delta \beta+\Delta M,
$$

where $\Delta \beta$ and $\Delta M$ are the gains in life expectancy resulting from changes in the shape parameter and modal age at death, respectively. In the following section we present the methodology of the decomposition for the Gompertz force of mortality and then generalize it to other parametric functions of mortality.

Figure 1: Illustration of the shifting and variability effects in the density function of the distribution of deaths for simulated data from a Gompertz model with a combination of shape parameters $\beta_{1}=0.10$ and $\beta_{2}=0.13$ and modal ages at death $M_{1}=75$ and $M_{2}=90$

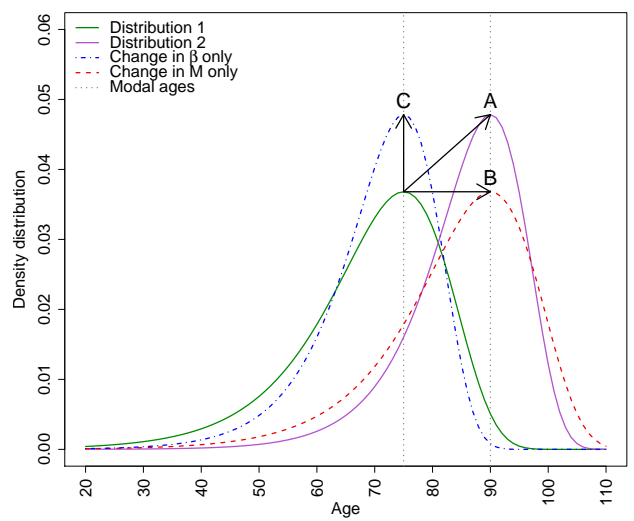




\subsection{Decomposing senescent mortality: Gompertz}

Gavrilov and Gavrilova (1991) defined the Gompertz (1825) law of mortality as one of the most successful models expressing mathematically the senescent age-pattern of mortality. In this article, we refer to senescent mortality as the increase over age in the force of mortality occuring after a certain age, representing aging and physiological deterioration (Bongaarts and Feeney 2002; Bongaarts 2005; Horiuchi et al. 2013). The Gompertz approach allows a good approximation of adult mortality patterns over age and time for many countries. However, the Gompertz model does not fit infant, child and oldest-old mortality well. Other parametric models, such as the Makeham (1860) and Siler (1979), have addressed some of these problems by including additional parameters capturing background and infant mortality. The Gompertz model is, however, broadly used to describe the distribution of adult death from age 30 to 90, having the advantage of being simple and offering a good fit to senescent mortality. The decomposition methodology introduced here will be presented through the Gompertz model, but it will be demonstrated that the method can be applied to other parametric models.

It has been shown by Horiuchi et al. (2013) and Missov et al. (2015) that the hazard rate as expressed by the Gompertz model can be rewritten using the modal age at death instead of the timing parameter $\alpha_{t}$ as

$$
\mu_{x, t}=\alpha_{t} e^{\beta_{t} x}=\beta_{t} e^{\beta_{t}\left(x-M_{t}\right)},
$$

where $\beta_{t}$ is the shape parameter at time $t$ of the Gompertz hazard function $\mu_{x, t}$, and $M_{t}$ is the modal age at death. This parametrization has some advantages: 1) the parameter $M_{t}$ has a clearer interpretation than $\alpha_{t}$ (Horiuchi et al. 2013), and 2) there is a lower correlation between the parameters when the Gompertz is expressed using the modal age at death (Missov et al. 2015).

The parametrization presented in equation (2) also gives a starting point for decomposing changes in life expectancy due to changes in variability and shifting mortality. Shifting mortality is observed through changes in the modal age at death, which is captured by the parameter $M_{t}$. Additionally, as presented in Appendix A, it can analytically be shown that the shape parameter $\beta_{t}$ is the main carrier of variability changes.

Let a dot on top of a variable denote its derivative with respect to time (Vaupel and Canudas-Romo 2003). The change over time in the force of mortality $\left(\dot{\mu}_{x, t}\right)$ can be decomposed into respective components of change for the shape $\left(\dot{\beta}_{t}\right)$ and the mode $\left(\dot{M}_{t}\right)$ :

$$
\dot{\mu}_{x, t}=\dot{\beta}_{t}\left[\mu_{x, t}\left(\frac{1}{\beta_{t}}+x-M_{t}\right)\right]-\dot{M}_{t}\left[\beta_{t} \mu_{x, t}\right] .
$$

Equation (3) can be expressed in more general terms to be used in other models of mortality. The components of change for the shape $\left(\dot{\beta}_{t}\right)$ and modal age at death $\left(\dot{M}_{t}\right)$ are each 
multiplied by a weighting function of the corresponding hazard rate, denoted as $f_{i}\left(\mu_{x, t}\right)$, with $i$ corresponding to the parameters $\beta$ and $M$,

$$
\dot{\mu}_{x, t}=\dot{\beta}_{t} f_{\beta}\left(\mu_{x, t}\right)-\dot{M}_{t} f_{M}\left(\mu_{x, t}\right) .
$$

As with the hazard distribution, we can derive the time change of life expectancy. In general terms, life expectancy at birth is expressed as

$$
e_{0, t}=\int_{0}^{\omega} l_{a, t} d a
$$

where $l_{a, t}$ is the survival function and the radix of the population is one. Therefore, changes in life expectancy at birth through time $\left(\dot{e}_{0, t}\right)$ can be expressed by:

$$
\dot{e}_{0, t}=\int_{0}^{\omega} i_{a, t} d a=-\int_{0}^{\omega} l_{a, t} \int_{0}^{a} \dot{\mu}_{x, t} d_{x} d_{a},
$$

where $i_{a, t}$ is the time derivative of the survival function $l_{a, t}$. By substituting equation (4) in equation (5), we can estimate the change in life expectancy at birth due to changes in the modal age at death and changes in the shape parameter as:

$$
\dot{e}_{0, t}=\underbrace{-\dot{\beta}_{t} \int_{0}^{\omega} l_{a, t} \int_{0}^{a} f_{\beta}\left(\mu_{x, t}\right) d_{x} d_{a}}_{\Delta \beta}+\underbrace{\dot{M}_{t} \int_{0}^{\omega} l_{a, t} \int_{0}^{a} f_{M}\left(\mu_{x, t}\right) d_{x} d_{a}}_{\Delta M} .
$$

The first term in equation (6) represents the gain in life expectancy resulting from a change in variability $(\Delta \beta)$, corresponding to a compression pattern, while the second term is the gain in life expectancy produced by a shift in the modal age at death $(\Delta M)$, indicating a shifting pattern. These are the equivalent terms of equation (1) in the Gompertz model.

Equations (4) and (6) allow further generalizations to other parametric models expressing senescent mortality using the modal age at death $(M)$ and a shape parameter $(\beta)$. Horiuchi et al. (2013) present this parametrization for the Logistic and Weibull models. Table 1 includes the elements of the decomposition equations for the Gompertz, Logistic and Weibull models.

As mentioned previously, the Gompertz model does not fit young age mortality well, and tends to fit mortality over age 30 better. Hence, for the application of the Gompertz decomposition, we will start our life table at age 30 , and $e_{0, t}$ will represent the life expectancy at age 30 , indicated as $e_{30, t}$ in the tables. 
Bergeron-Boucher, Ebeling \& Canudas-Romo: Decomposing changes in life expectancy

Table 1: $\quad$ Hazard $\left(\mu_{x}\right)$, modal age at death $(M)$, life expectancy at birth $\left(e_{0}\right)$ and decomposition weights $\left(f_{\beta}\left(\mu_{x, t}\right)\right.$ and $\left.f_{M}\left(\mu_{x, t}\right)\right)$ for three mortality models

Gompertz $\quad$ Logistic $\quad$ Weibull

\begin{tabular}{cccc}
\hline $\boldsymbol{\mu}_{\boldsymbol{x}}(\boldsymbol{\alpha}, \boldsymbol{\beta})$ & $\alpha e^{\beta x}$ & $\frac{e^{\alpha+\beta x}}{1+e^{\alpha+\beta x}}$ & $\alpha \beta x^{\beta-1}$ \\
$\boldsymbol{\mu}_{\boldsymbol{x}}(\boldsymbol{M}, \boldsymbol{\beta})$ & $\beta e^{\beta(x-M)}$ & $\frac{\beta e^{\beta(x-M)}}{1+\beta e^{\beta(x-M)}}$ & $\frac{(\beta-1) x^{\beta-1}}{(M)^{\beta}}$ \\
\hline $\boldsymbol{M}$ & $\frac{\ln (\beta)-\ln (\alpha)}{\beta}$ & $\frac{\ln (\beta)-\alpha}{\beta}$ & $\left(\frac{(\beta-1)}{\alpha \beta}\right)^{\frac{1}{\beta}}$ \\
\hline $\boldsymbol{e}_{\mathbf{0}}$ & $\int_{0}^{\omega} e^{-e^{-\beta M}\left(e^{\beta a}-1\right)} d a$ & $\int_{0}^{\omega}\left[\frac{\beta e^{-\beta M}+1}{\beta e^{\beta(a-M)}+1}\right]^{\frac{1}{\beta}} d a$ & $\int_{0}^{\omega} e^{-\frac{(\beta-1) a^{\beta}}{\beta M^{\beta}}} d a$ \\
\hline $\boldsymbol{f}_{\boldsymbol{\beta}(\boldsymbol{\mu} \boldsymbol{x}, \boldsymbol{t})}$ & $\mu_{x, t}\left(\frac{1}{\beta_{t}}+x-M_{t}\right)$ & $\frac{\mu_{x, t}\left(\frac{1}{\beta_{t}}+x-M_{t}\right)}{1+\beta_{t} e^{\beta}\left(x-M_{t}\right)}$ & $\mu_{x, t}\left[\frac{1}{\left(\beta_{t}-1\right)}+\ln \left(\frac{x}{M_{t}}\right)\right]$ \\
\hline $\boldsymbol{f}_{\boldsymbol{M}}\left(\boldsymbol{\mu}_{\boldsymbol{x}, \boldsymbol{t}}\right)$ & $\beta_{t} \mu_{x, t}$ & $\frac{\beta_{t} \mu_{x, t}}{1+\beta_{t} e^{\beta\left(x-M_{t}\right)}}$ & $\mu_{x, t} \frac{\beta_{t}}{M_{t}}$
\end{tabular}

Note: To simplify the equations, the time component (t) was not added as subscript to the parameters $\alpha_{t}, \beta_{t}$ and $M_{t}$ in the first four lines of the table. However, the parameters can also vary over time (t) in these equations.

\subsection{Extending the model beyond senescent mortality}

With the previous methodology, only senescent mortality can be decomposed. The decomposition is thus limited to adult and old-age mortality, and might bring only limited understanding of mortality changes over time. As mentioned previously, compression of mortality has been strongly linked to reductions in infant, child and early adult mortality, which is not considered when decomposing the Gompertz model. Modeling mortality at all ages needs more complex models, and additional parameters often need to be added.

Equation (1) can be generalized to allow the inclusion of parameters other than $\beta$ and $M$, as

$$
\dot{e}_{0, t}=\sum_{i=1} \Delta_{i}
$$


where $\Delta_{i}$ is the change in life expectancy at birth due to a change in the parameter $i$.

\subsubsection{Gompertz-Makeham}

A Makeham (1860) variant can be added to each of the models presented in Table 1 (Horiuchi et al. 2013). Assuming that the modal age at death $\left(M_{t}\right)$ estimated by the Gompertz model in equation (2) applies to the Gompertz-Makeham model, the hazard function can be expressed as

$$
\mu_{x, t}=c_{t}+\beta_{t} e^{\beta_{t}\left(x-M_{t}\right)},
$$

where $c_{t}$ is the Makeham term. Adding the parameter $c_{t}$ improves the fit of the Gompertz function at younger ages, but still without capturing the decrease in infant mortality. The Makeham term is an age-independent component which captures the extrinsic or "background" mortality risk. The Makeham term has a more influential effect at younger ages and is often associated with adult or early adult mortality, which is especially important for the variability effect.

Equivalent to the decomposition presented in equation (6), we can estimate the change in adult life expectancy due to changes in the different parameters of the GompertzMakeham model using equation (5). As expressed by equation (7), change in life expectancy is then estimated as

$$
\begin{aligned}
\dot{e}_{0, t}= & -\underbrace{\dot{c}_{t} \int_{0}^{\omega} l_{a, t} a d a}_{\Delta c}-\underbrace{\dot{\beta}_{t} \int_{0}^{\omega} l_{a, t} \int_{0}^{a}\left[e^{\beta_{t}\left(x-M_{t}\right)}\left(1+\beta_{t}\left(x-M_{t}\right)\right)\right] d x d a}_{\Delta \beta} \\
& +\underbrace{\dot{M}_{t} \int_{0}^{\omega} l_{a, t} \int_{0}^{a}\left[\beta_{t}^{2} e^{\beta_{t}\left(x-M_{t}\right)}\right] d x d a}_{\Delta M},
\end{aligned}
$$

where $\dot{c}_{t}$ is the change in the background mortality level, $\dot{\beta}_{t}$ is the change in the rate of mortality increase over age and $\dot{M}_{t}$ is the change in the modal age at death.

\subsubsection{Siler}

The Siler (1979) model extends the Gompertz model by including two additional terms, capturing both the decrease over ages of infant mortality and the "background" mortality risk. By using the Gompertz model with the parametrization presented in equation (2), we can express the Siler model as 


$$
\mu_{x, t}=\alpha_{t} e^{-b_{t} x}+c_{t}+\beta_{t} e^{\beta_{t}\left(x-M_{t}\right)},
$$

where $\alpha_{t}$ and $c_{t}$ are timing parameters for infant and background mortality, the parameters $b_{t}$ and $\beta_{t}$ are the constant rates of mortality change over age for infant and senescent mortality, respectively, and $M_{t}$ is the modal age at death. By including the infant and background parameters, the Siler model provides a more detailed estimation of the variability and shifting effect by modeling mortality at all ages.

Decomposition of changes in the Siler model is expressed by changes in 5 different parameters: $\dot{\alpha}_{t}$ is the change with respect to $t$ in the initial level of mortality (age 0 ), $\dot{b}_{t}$ is the change in the rate of infant mortality decrease over age, $\dot{c}_{t}$ is the change in the background mortality level, $\dot{\beta}_{t}$ is the change in the rate of mortality increase over age for senescent mortality, and $\dot{M}_{t}$ is the change in the modal age at death.

As generally presented in equation (7), the gain in life expectancy at birth for the Siler model is estimated by

$$
\begin{aligned}
\dot{e}_{0, t}= & -\underbrace{\dot{\alpha}_{t} \int_{0}^{\omega} l_{a, t} \int_{0}^{a}\left[e^{-b_{t} x}\right] d x d a}_{\Delta \alpha}+\underbrace{\dot{b}_{t} \int_{0}^{\omega} l_{a, t} \int_{0}^{a}\left[\alpha_{t} e^{-b_{t} x} x\right] d x d a}_{\Delta b} \\
& -\underbrace{\dot{c}_{t} \int_{0}^{\omega} l_{a, t} a d a}_{\Delta c}-\underbrace{\dot{\beta}_{t} \int_{0}^{\omega} l_{a, t} \int_{0}^{a}\left[e^{\beta_{t}\left(x-M_{t}\right)}\left(1+\beta_{t}\left(x-M_{t}\right)\right)\right] d x d a}_{\Delta M} \\
& +\underbrace{}_{\Delta \int_{0} \operatorname{M}_{t} \int_{a, t} \int_{0}^{a}\left[\beta_{t}^{2} e^{\beta_{t}\left(x-M_{t}\right)}\right] d x d a} .
\end{aligned}
$$

There are, however, some implications of adding the Makeham term and the Siler infant mortality term to the Gompertz parametrization presented in equation (2). The parameter $M$, reflecting the modal age at death evaluated from senescent mortality only, could differ from the modal age at death evaluated from the total mortality. Horiuchi et al. (2013), however, found that the modal age at death for senescent mortality, when adding a Makeham term to equation (2), is nearly equal to the modal age at death for total mortality. We found similar results with the Siler model. For example, the total modal age at death for Swedish female mortality in 2010 fitted with a Siler model was 88.46, and the senescent modal age at death was 88.49. As the mortality level at old-ages tends to be determined by senescent mortality, with only limited influence from infant and background mortality, the senescent and total modal ages at death will generally be similar (Horiuchi et al. 2013). It is important to recall that the modal age at death is determined by old-age mortality only. To help understand the role of young age mortality on shifting and compression, we decided to overlook these small differences. In the following sections, we 
will refer to the senescent modal age at death as the modal age at death.

When using the Gompertz model, the variability effect is captured by the parameter $\beta_{t}$ (Appendix A) and the shifting effect by the parameter $M_{t}$. However, with a GompertzMakeham model or a Siler model, more parameters will influence variability changes. Canudas-Romo (2010) analytically demonstrated that, in a mortality declining scenario, as the one experienced in developed countries, the mode will be maintained when reduction of mortality occurs at younger ages than the modal age at death. Using a Siler model, Engelman, Caswell, and Agree (2014) showed that improvement in childhood components of mortality $\left(\alpha_{t}\right.$ and $\left.b_{t}\right)$ and in background mortality parameter $\left(c_{t}\right)$ influenced lifespan variability reduction. The first four terms of the above equation would then have an impact on variability reduction. The variability effect could then be divided into four distinct effects: $\dot{\alpha}_{t}, \dot{b}_{t}, \dot{c}_{t}$ and $\dot{\beta}_{t}$. The shifting effect is still captured by $\dot{M}_{t}$. This partition between the five Siler parameters emphasizes the impact of changing mortality at young ages on lifespan disparities, in contrast with the effect of mortality reductions at older ages on shifting mortality.

\subsection{Data}

The data source used in this study is the Human Mortality Database (HMD: http://www. mortality.org). The HMD (2015) compiles census and vital statistics information for the populations of entire countries. The HMD has high quality historical mortality data for industrialized countries; the data series are constructed according to a common protocol, making the HMD a unique comparison tool. For our illustrations, data for all the HMD countries, excluding Eastern European countries, have been used for years 1900 to 2010 (Table 2). We justify the data exclusion because there are different age-patterns of mortality in the excluded countries than to those included in the illustrations in recent decades. Nevertheless, our methodology can easily be extended to those countries although with different mortality parameters.

The decomposition is applied to the mortality of Swedish females and to the average female mortality in the selected HMD countries ${ }^{4}$. The Gompertz, Gompertz-Makeham and Siler models are fitted to observed mortality trends using a Poisson log-likelihood procedure. The estimation procedures of derivatives such as those in equations (6) to discrete data are presented in Appendix B.

\footnotetext{
${ }^{4}$ The parameters of the mortality models are estimated for each country independently and then averaged over all countries (with equal weight) to obtain the HMD average.
} 
Table 2: $\quad$ Selected HMD countries and years with available data used for the illustration

\begin{tabular}{llll}
\hline Country & Years & Country & Years \\
\hline Australia & $1925-2010$ & Japan & $1947-2010$ \\
Austria & $1947-2010$ & Luxembourg & $1960-2009$ \\
Belgium & $1900-2010$ & Netherlands & $1900-2009$ \\
Canada & $1921-2010$ & New Zealand & $1948-2008$ \\
Chile & $1992-2005$ & Norway & $1900-2009$ \\
Denmark & $1900-2010$ & Portugal & $1940-2010$ \\
Finland & $1900-2010$ & Spain & $1908-2010$ \\
France & $1900-2010$ & Sweden & $1900-2010$ \\
Germany & $1990-2010$ & Switzerland & $1900-2010$ \\
Iceland & $1900-2010$ & Taiwan & $1970-2010$ \\
Ireland & $1950-2009$ & United Kingdom & $1922-2010$ \\
Israel & $1983-2009$ & United States & $1933-2010$ \\
Italy & $1900-2009$ & & \\
\hline
\end{tabular}

Source: HMD (2015)

\section{Illustration}

\subsection{Gompertz decomposition}

Table 3 presents the decomposition of life expectancy at age 30 by $M$ and $\beta$ for Swedish females at the beginning, middle, and end of the 20th century and for the HMD females average, between 2000 and 2005. For the three periods selected and for both populations, changes in the modal age at death $(\Delta M)$ are the main components driving the change in life expectancy.

To further study the year-to-year changes, Figure 2 presents the decomposition from 1900 until 2010 in 5-year intervals for Swedish and HMD average females. Over most periods, the gains in life expectancy at age 30 were mainly the result of a shift in the modal age at death $(\Delta M)$. Until the end of the 1950s, variability reduction contributions to changes in life expectancy $(\Delta \beta)$ have been more important than in the following periods. However, even during those years, changes in life expectancy were mainly driven by changes in the mode. Figure 7 in Appendix C presents similar results for 25 of the HMD countries. 
Table 3: $\quad$ Female life expectancy at age $30\left(e_{30, t}\right)$ and its decomposition due to changes in the Gompertz parameters, Sweden and HMD average, 1900, 1950, and 2000

\begin{tabular}{rccccc}
\hline & \multicolumn{3}{c}{ Sweden } & \multicolumn{2}{c}{ HMD Average } \\
& 1900 & 1950 & 2000 & 2000 & $(\min , \max )$ \\
\hline$e_{30, t}$ & 37.82 & 44.18 & 52.04 & 51.43 & $(49.23,54.79)$ \\
$e_{30, t+5}$ & 38.21 & 45.56 & 52.72 & 52.45 & $(50.40,55.67)$ \\
$\dot{e}_{30, t}$ & 0.39 & 1.39 & 0.68 & 1.03 & \\
& & & & & \\
$\Delta \beta$ & -0.17 & 0.09 & 0.05 & 0.09 & $(-0.11,0.22)$ \\
$\Delta M$ & 0.56 & 1.30 & 0.63 & 0.94 & $(0.48,2.08)$ \\
$\Delta \beta+\Delta M$ & 0.39 & 1.39 & 0.68 & 1.03 & \\
\hline
\end{tabular}

Source: HMD (2015) and authors' own calculation.

Note: By rounding the numbers to the second decimal point in the table, the sum of the contributions $\left(\sum \Delta_{i}\right)$ might differ slightly from the difference in life expectancy $\left(\dot{e}_{30, t}\right)$.

Figure 2: $\quad$ Trends over time of the Gompertz parameters' contribution to changes in female life expectancy at age $30\left(\dot{e}_{30, t}\right)$, Sweden and HMD average, 1900-2010

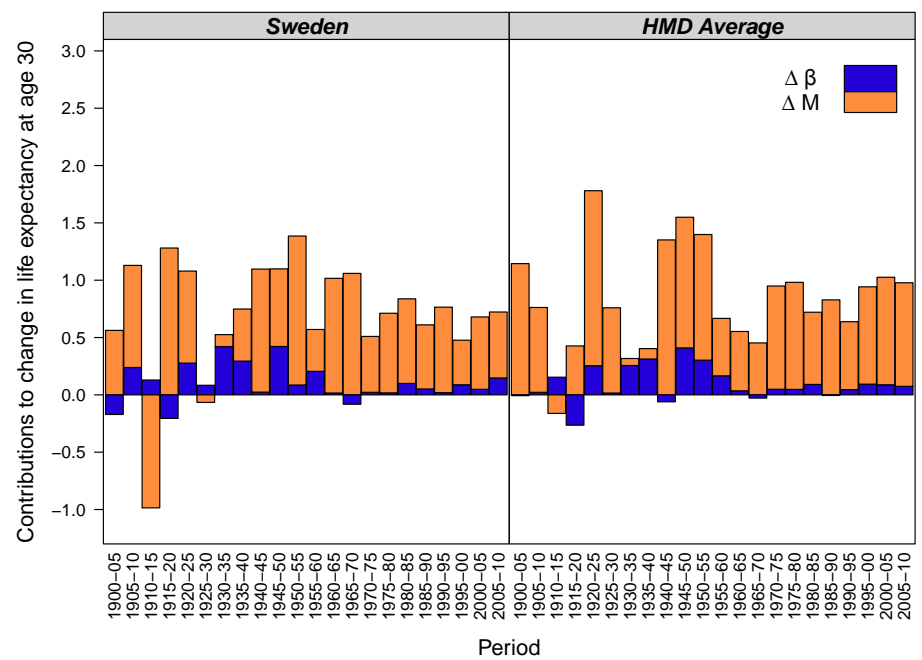

Source: HMD (2015) and authors' own calculation. 
Changes in variability of senescent mortality alone would not have been sufficient to generate the important gains in life expectancy at age 30 observed since 1900. An explanation for this small variability effect compared with the important shifting effect for senescent mortality still needs to be provided. A possible explanation is that as only senescent mortality is analyzed by the Gompertz model, it does not consider the ages essentially responsible for mortality compression, i.e., infant, child and early adult (Cheung and Robine 2007). To address the latter aspect of how mortality at young ages has influenced changes in life expectancy, we present results for the Gompertz-Makeham and Siler models in the next sections.

\subsection{Gompertz-Makeham decomposition}

The Gompertz-Makeham model can help us understand the impact of early adult mortality changes on compression and shifting mortality. Table 4 presents an application of the decomposition of life expectancy at age 30 using the Gompertz-Makeham model for Swedish and HMD average females at three points in time. Among the parameters influencing variability changes ( $\beta$ and $c$ ), the Makeham term $(c)$ has a similar influence on life expectancy changes than the shape parameter $\beta$, for most of the times studied in Table 4 .

Table 4: $\quad$ Female life expectancy at age $30\left(e_{30, t}\right)$ and its decomposition due to changes in the Gompertz-Makeham parameters, Sweden and HMD average, 1900, 1950, and 2000

\begin{tabular}{rccccc}
\hline & \multicolumn{3}{c}{ Sweden } & \multicolumn{2}{c}{ HMD Average } \\
& 1900 & 1950 & 2000 & 2000 & $(\min , \max )$ \\
\hline$e_{30, t}$ & 32.50 & 43.30 & 51.41 & 50.75 & $(49.02,53.66)$ \\
$e_{30, t+5}$ & 32.95 & 45.04 & 52.09 & 51.70 & $(49.52,54.49)$ \\
$\dot{e}_{30, t}$ & 0.45 & 1.74 & 0.68 & 0.95 & \\
& & & & & \\
$\Delta c$ & 0.11 & 0.77 & 0.02 & -0.09 & $(-0.73,0.63)$ \\
$\Delta \beta$ & -0.12 & -0.03 & 0.05 & 0.11 & $(0.00,0.36)$ \\
$\Delta M$ & 0.46 & 1.00 & 0.61 & 0.93 & $(0.48,2.01)$ \\
$\Delta c+\Delta \beta+\Delta M$ & 0.45 & 1.74 & 0.68 & 0.95 & \\
\hline
\end{tabular}

Source: HMD (2015) and authors' own calculation.

Note: By rounding the numbers to the second decimal point in the table, the sum of the contributions $\left(\sum \Delta_{i}\right)$ might differ slightly from the difference in life expectancy $\left(\dot{e}_{30, t}\right)$.

Figure 3 presents the decomposition for 5-year periods between 1900 and 2010 for Swedish and HMD average females. The gains in life expectancy at age 30 before the 1950 s were mainly driven by changes in variability of the age at death, which is essentially captured 
by changes in the parameter $c$. After this initial period of variability decline, changes in life expectancy at age 30 are mainly the result of shifting mortality $(\Delta M)$.

The inclusion of a parameter capturing early adult background mortality appears essential, then, to demonstrate the effect of variability reduction on life expectancy at age 30. Figure 8 in Appendix $\mathrm{C}$ shows similar results for the selected HMD countries. The next section presents an application of the Siler decomposition, in order to understand the role of infant mortality on the changes in life expectancy.

Figure 3: $\quad$ Trends over time of the Gompertz-Makeham parameters' contribution to changes in female life expectancy at age $30\left(\dot{e}_{30, t}\right)$, Sweden and HMD average, 1900-2010

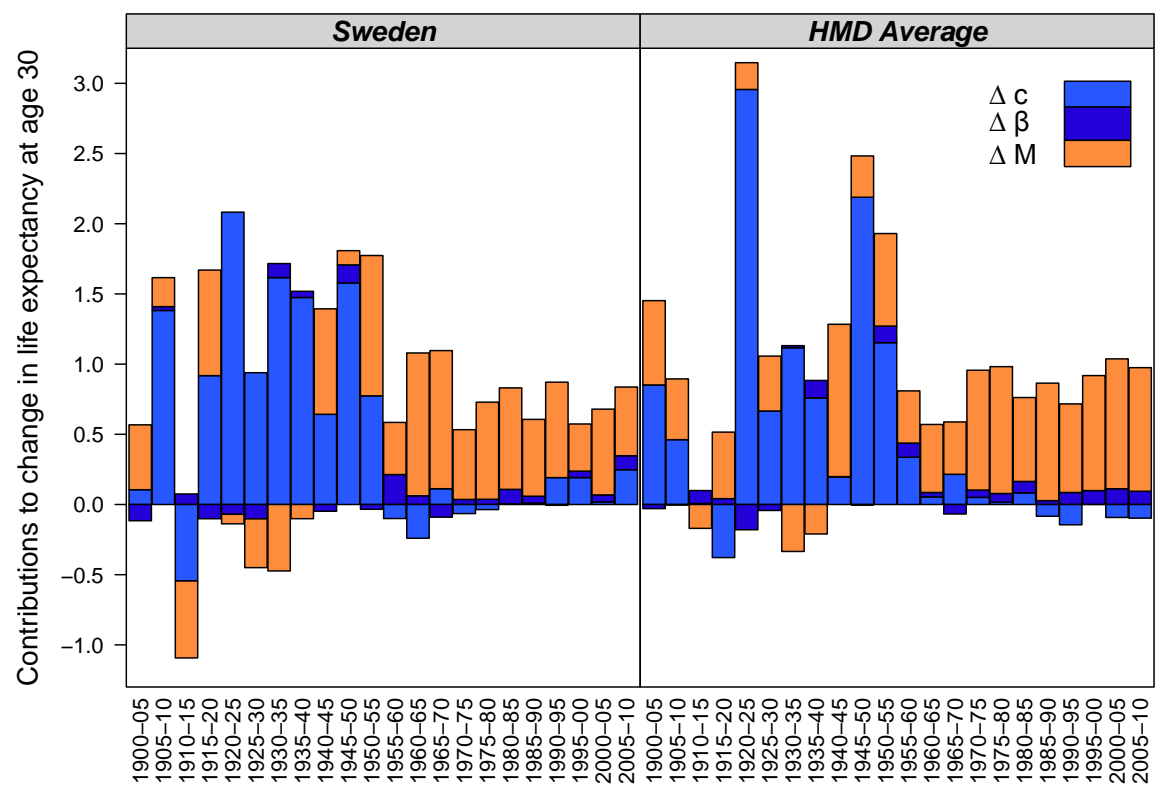

Period

Source: HMD (2015) and authors' own calculation.

\subsection{Siler decomposition}

Table 5 and Figure 4 present the results of life expectancy decomposition, using a Siler model, for female mortality in Sweden and HMD average at ages 0 and older. Similar 
to the Gompertz-Makeham decomposition, the results suggest that changes in life expectancy at birth before the 1950s were mainly the result of variability reductions. Gains in life expectancy due to changes in the parameter $\beta$ are still small, and the main gains are due to variability reductions coming from changes in infant and background parameters.

Since the mid-1960s, the modal age at death has been the key parameter leading the changes in life expectancy. Changes in the modal age at death were responsible for more than $70 \%$ of the increase in $e_{0, t}$ since 1965 for females from both Swedish and HMD average. Figure 9 in Appendix C presents similar results for 25 of the HMD countries.

Table 5: $\quad$ Female life expectancy at age $0\left(e_{0, t}\right)$ and its decomposition due to changes in the Siler parameters, Sweden and HMD average, 1900, 1950 and 2000

\begin{tabular}{rccccc}
\hline & \multicolumn{3}{c}{ Sweden } & \multicolumn{2}{c}{ HMD Average } \\
& 1900 & 1950 & 2000 & 2000 & $(\min , \max )$ \\
\hline$e_{0, t}$ & 56.06 & 72.78 & 81.68 & 80.93 & $(78.92,84.16)$ \\
$e_{0, t+5}$ & 58.00 & 74.44 & 82.34 & 81.99 & $(79.92,85.01)$ \\
$\dot{e}_{0, t}$ & 1.94 & 1.66 & 0.66 & 1.06 & \\
& & & & & \\
$\Delta \alpha$ & 0.52 & 0.06 & 0.01 & 0.01 & $(-0.04,0.04)$ \\
$\Delta b$ & 0.54 & 0.02 & -0.04 & 0.00 & $(-0.07,0.05)$ \\
$\Delta c$ & 0.69 & 0.44 & 0.02 & 0.04 & $(-0.16,0.23)$ \\
$\Delta \beta$ & -0.17 & 0.03 & 0.05 & 0.09 & $(-0.07,0.26)$ \\
$\Delta M$ & 0.34 & 1.11 & 0.62 & 0.91 & $(0.48,2.01)$ \\
$\Delta \alpha+\Delta b+\Delta c+\Delta \beta+\Delta M$ & 1.94 & 1.66 & 0.66 & 1.06 & \\
\hline
\end{tabular}

Source: HMD (2015) and authors' own calculation.

Note: By rounding the numbers to the second decimal point in the table, the sum of the contributions $\left(\sum \Delta_{i}\right)$ might differ slightly from the difference in life expectancy $\left(\dot{e}_{0, t}\right)$. 
Figure 4: $\quad$ Trends over time of the Siler parameters' contribution to changes in female life expectancy at age $0\left(\dot{e}_{0, t}\right)$, Sweden and HMD average, 1900-2010

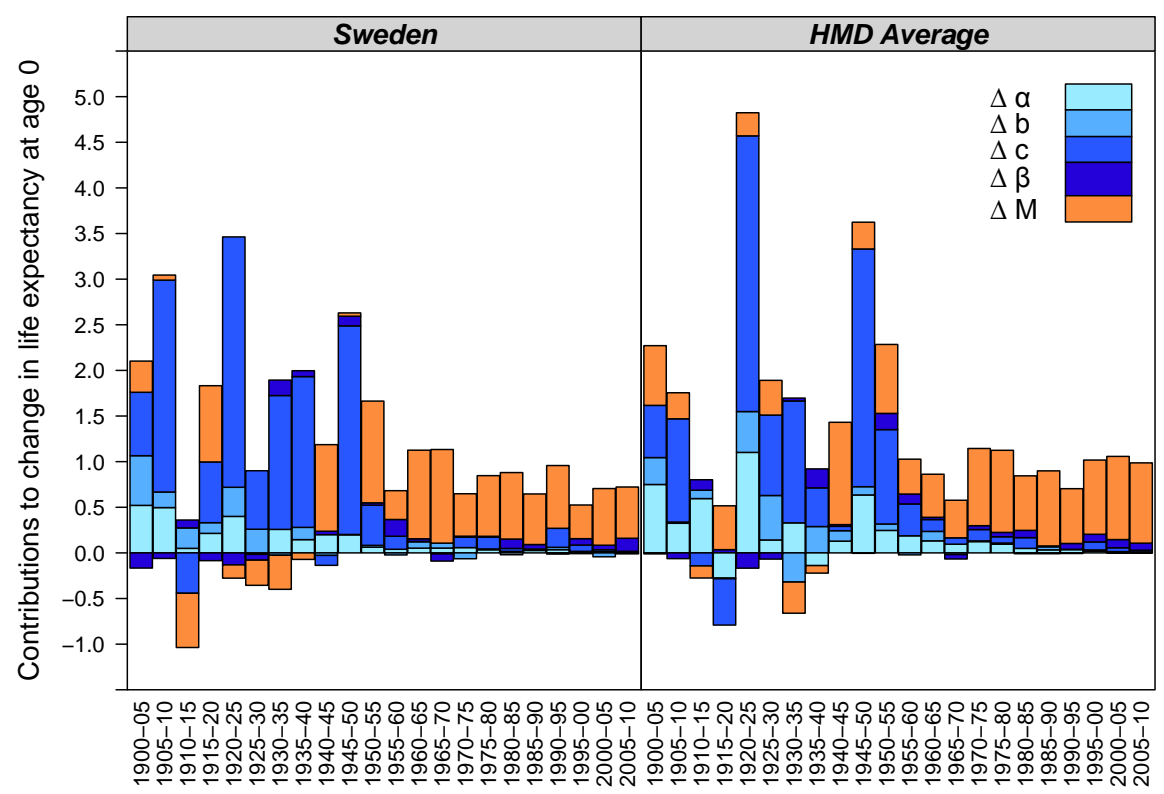

Period

Source: HMD (2015) and authors' own calculation.

Note: Appendix D presents these results in terms of relative differences.

\subsection{Life expectancy and modal age at death}

Figure 5 shows the life expectancy at birth and the modal age at death between 1900 and 2010 for females from Sweden and HMD average. Until the beginning of the 1940s, life expectancy increased around 4 months per year on average, while the modal age at death stayed nearly constant. The gains in life expectancy over this first period were in great part the result of improvements in infant and background mortality, and thus, variability reductions (Figure 4). 


\section{Figure 5: $\quad$ Modal age at death and life expectancy at birth and their respective segmented regression for females, Sweden and HMD average, 1900-2010}

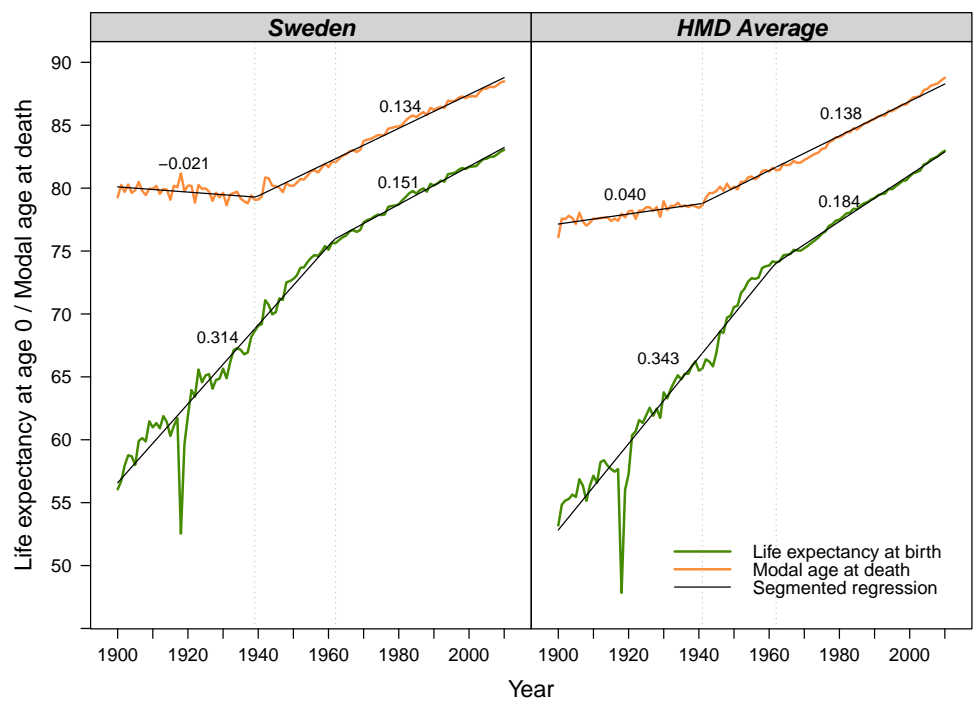

Source: HMD (2015) and authors' own calculation.

Note: The slopes and breakpoints of the modal age at death and life expectancy trends are calculated with a segmented regression methodology (Camarda, Vallin, and Meslé 2012).

A second period followed in which the mode begins to increase at a faster pace while life expectancy increase keeps its previous pace. Between 1940 and 1965, the mode contribution to changes in life expectancy increased, while variability contributions gradually lost importance. During these years, variability contributions to changes in life expectancy decreased from more than $60 \%$ to less than $30 \%$ of the total gains (Appendix D). This period is one of acceleration in the increase of the modal age at death and marks the transition from compression to shifting mortality.

Since the mid-1960s, the mode and life expectancy have been increasing at a comparable pace of around 2 months per year. This change in the pace of life expectancy coincides with the change of pace observed by Vallin and Meslé (2009) for the bestpractice life expectancy. Life expectancy is still increasing at a marginally faster pace than the mode due to small extra contributions from variability reduction. Nevertheless, gains in life expectancy are mainly driven by changes in the modal age at death and thus shifting mortality (Figure 4). This parallels the 3-phases transition described by Cheung et al. (2009). 


\section{Discussion and conclusion}

Using recent parametrization of the Gompertz model, we separated changes in life expectancy by the variability reduction effect, captured by $\beta$, from the shifting mortality effect, captured by $M$. The methodology is then extended to other parametric representations of mortality, and particularly to the Gompertz-Makeham and Siler models, to consider the effect of young adult, child and infant mortality changes. This new decomposition method, using parametric models, allows us to understand and quantify the respective impact of shifting mortality and variability changes on life expectancy.

Our results suggest that mortality compression was the main driver of change in life expectancy at birth before the 1950s, due to a decrease in infant and background mortality. After this period, changes in life expectancy became gradually dependent on the shift in the senescent modal age at death. These results are consistent with the findings of other studies looking at changes in the modal age at death and at different variability measures (Wilmoth and Horiuchi 1999; Robine 2001; Yashin et al. 2001; Canudas-Romo 2008). The results also confirm the increasing importance of the modal age at death as a key indicator of lifespan. The modal age at death has increased since the beginning of the 1940s and has become the main driver of longevity extension since the 1960s. An important feature of this indicator is that, in populations that experience declining mortality, its change is only determined by old-age mortality.

In the above illustrations, the results of the decomposition are presented for female life expectancy only. However, similar results are found when decomposing male life expectancy, but with a shifting pattern appearing later in time. Shifting mortality became the main driver of life expectancy increase in the late 1970s for males (results available from the authors).

We asked previously how and to what extent, one process replaced the other. Our methodology allows us to observe and quantify the gradual replacement of a compression pattern by a shifting pattern in a relatively short period of time. We can also observe that, even if shifting the modal age at death is explaining a great deal of the life expectancy increase nowadays, lifespan variability reductions still play a role in mortality changes.

The results are, however, sensitive to the selected parametric model, and especially if the model is able to include infant and background mortality parameters. The dominating effect of variability reductions on life expectancy increase in the first half of the 20th century can only be seen when using the Gompertz-Makeham and Siler models in the above illustrations. When only senescent mortality is analyzed, the compression of mortality only has a minor impact on life expectancy improvement, even before the 1950s. It could thus be theorized that in a context where infant and young adult mortality is low, as it is the case in most industrialized countries, variability reductions will have limited impact on life expectancy, and shifting the modal age at death would be the main dynamic that would allow life expectancy to increase. 
The choice of the parametric model for senescent mortality might also have influenced the results. The illustration section presented the application of the methodology to discrete data using a Gompertz model. Other models could, however, have been more appropriate, such as the Logistic, to consider the deceleration in the hazard at very oldages. However, an application using the Logistic model shows that the results are very similar to the findings obtained with the Gompertz model (Appendix E).

A previous attempt to quantify the effect of shifting the mortality schedule on life expectancy has been done by De Beer and Janssen (2014). Their procedure consists in evaluating the effect on life expectancy of changing the value of their model parameters on life expectancy. However, to the authors' knowledge, our current study is the first attempt to quantify the gain in life expectancy produced by a change in variability and shifting mortality. Our procedure allows us to differentiate between both underlying mortality processes and their respective impact on life expectancy, and also to determine when and how one process has replaced the other.

\section{Acknowledgements}

The authors are grateful to Jim Vaupel, Jim Oeppen, Adam Lenart, Virginia Zarulli and the rest of the Max-Planck Odense Center on the Biodemography of Aging for their useful comments, suggestions and support. We also thank Samir Soneji, Roland Rau, Christina Bohk-Ewald and Frederik Peters for useful comments on earlier versions of this work. We are also thankful to the two anonymous reviewers for their helpful and constructive comments, as well as Iris Levitis and Kirsten Gauthier for the English editing. The third author would like to thank the European-Research-Council grant-240795 for supporting his work on this project. 


\section{References}

Andreev, E.M., Shkolnikov, V.M., and Begun, A.Z. (2002). Algorithm for decomposition of differences between aggregate demographic measures and its application to life expectancies, healthy life expectancies, parity-progression ratios and total fertility rates. Demographic Research 7(14): 499-522. doi:10.4054/DemRes.2002.7.14.

Arriaga, E. (1984). Measuring and explaining the change in life expectancies. Demography 21(1): 83-96. doi:10.2307/2061029.

Beltrán-Sánchez, H., Preston, S., and Canudas-Romo, V. (2008). An integrated approach to cause-of-death analysis: cause-deleted life tables and decompositions of life expectancy. Demographic Research 19(35): 1323-1350. doi:10.4054/DemRes.2008.19.35.

Bongaarts, J. (2005). Long-range trends in adult mortality: Models and projection methods. Demography 42(1): 23-49. doi:10.1353/dem.2005.0003.

Bongaarts, J. and Feeney, G. (2002). How long do we live? Population and Development Review 28(1): 13-29. doi:10.1111/j.1728-4457.2002.00013.x.

Camarda, G., Vallin, J., and Meslé, F. (2012). Identifying the ruptures shaping the segmented line of secular trends in maximum life expectancies. In: European Population Conference 2012: Gender, Policies and Population.

Canudas-Romo, V. (2008). The modal age at death and the shifting mortality hypothesis. Demographic Research 19(30): 1179-1204. doi:10.4054/DemRes.2008.19.30.

Canudas-Romo, V. (2010). Three measures of longevity: Time trends and record values. Demography 47(2): 299-312. doi:10.1353/dem.0.0098.

Cheung, S.L.K. and Robine, J.M. (2007). Increase in common longevity and the compression of mortality: The case of Japan. Population Studies 61(1): 85-97. doi:10.1080/00324720601103833.

Cheung, S.L.K., Robine, J.M., Paccaud, F., and Marazzi, A. (2009). Dissecting the compression of mortality in Switzerland, 1876-2005. Demographic Research 21(19): 569598. doi:10.4054/DemRes.2009.21.19.

Cheung, S.L.K., Robine, J.M., Tu, E.J.C., and Caselli, G. (2005). Three dimensions of the survival curve: Horizontalization, verticalization, and longevity extension. Demography 42(2): 243-258. doi:10.1353/dem.2005.0012.

Christensen, K., Doblhammer, G., Rau, R., and Vaupel, J.W. (2009). Ageing populations: the challenges ahead. The Lancet 374(9696): 1196 - 1208. doi:10.1016/S01406736(09)61460-4. 
De Beer, J. and Janssen, F. (2014). The NIDI mortality model; a new model to describe the age pattern of mortality. NIDI, Working Paper 2014/7.

Edwards, R.D. and Tuljapurkar, S. (2005). Inequality in life spans and a new perspective on mortality convergence across industrialized countries. Population and Development Review 31(4): 645-674. doi:10.1111/j.1728-4457.2005.00092.x.

Engelman, M., Canudas-Romo, V., and Agree, E.M. (2010). The implications of increased survivorship for mortality variation in aging populations. Population and Development Review 36(3): 511-539. doi:10.1111/j.1728-4457.2010.00344.x.

Engelman, M., Caswell, H., and Agree, E. (2014). Why do lifespan variability trends for the young and old diverge? A perturbation analysis. Demographic Research 30(48): 1367-1396. doi:10.4054/DemRes.2014.30.48.

Firebaugh, G., Acciai, F., Noah, A.J., Prather, C.J., and Nau, C. (2014). Why the racial gap in life expectancy is declining in the United States. Demographic Research 31(32): 975-1006. doi:10.4054/DemRes.2014.31.32.

Fries, J.F. (1980). Aging, natural death, and the compression of morbidity. New England Journal of Medicine 303(3): 130-135. doi:10.1056/NEJM198007173030304.

Gavrilov, L.A. and Gavrilova, N.S. (1991). The biology of life span: a quantitative approach. Chur, Switzerland: Harwood Academic Publications.

Gompertz, B. (1825). On the nature of the function expressive of the law of human mortality, and on a new mode of determining the value of life contingencies. Philosophical Transactions of the Royal Society of London 115: 513-583. doi:10.1098/rstl.1825.0026.

HMD (2015). Human Mortality Database, University of California, Berkeley (USA), and Max Planck Institute for Demographic Research (Germany). http://www.mortality.org.

Horiuchi, S., Ouellette, N., Cheung, S.L.K., and Robine, J.M. (2013). Modal age at death: lifespan indicator in the era of longevity extension. Vienna Yearbook of Population Research 11: 37-69. doi:10.1553/populationyearbook2013s37.

Horiuchi, S., Wilmoth, J., and Pletcher, S. (2008). A decomposition method based on a model of continuous change. Demography 45(4): 785-801. doi:10.1353/dem.0.0033.

Kannisto, V. (2000). Measuring the compression of mortality. Demographic Research 3(6): 24. doi:10.4054/demres.2000.3.6.

Kannisto, V. (2001). Mode and dispersion of the length of life. Population: An English Selection 13(1): pp. 159-171. http://www.jstor.org/stable/3030264.

Keyfitz, N. (1977). What difference would it make if cancer were eradicated? An exami- 
nation of the taeuber paradox. Demography 14(4): 411-418. doi:10.2307/2060587.

Makeham, W.M. (1860). On the law of mortality and the construction of annuity tables. The Assurance Magazine, and Journal of the Institute of Actuaries 8(6): 301-310. http://www.jstor.org/stable/41134925.

Missov, T.I., Lenart, A., Nemeth, L., Canudas-Romo, V., and Vaupel, J.W. (2015). The Gompertz force of mortality in terms of the modal age at death. Demographic Research 32(36): 1031-1048. doi:10.4054/DemRes.2015.32.36.

Nusselder, W.J. and Mackenbach, J.P. (1996). Rectangularization of the survival curve in the Netherlands, 1950-1992. The Gerontologist 36(6): 773-782. doi:10.1093/geront/36.6.773.

Oeppen, J. and Vaupel, J.W. (2002). Broken limits to life expectancy. Science 296(5570): 1029-1031. doi:10.1126/science.1069675.

Ouellette, N. and Bourbeau, R. (2011). Changes in the age-at-death distribution in four low mortality countries: A nonparametric approach. Demographic Research 25(19): 595-628. doi:10.4054/DemRes.2011.25.19.

Pollard, J.H. (1982). The expectation of life and its relationship to mortality. Journal of the Institute of Actuaries 109: 225-240. doi:10.1017/S0020268100036258.

Pressat, R. (1985). Contribution des écarts de mortalité par âge à la différence des vies moyennes. Population (French Edition) 40(4-5): 766-770. doi:10.2307/1532986.

Preston, S., Heuveline, P., and Guillot, M. (2001). Demography: Measuring and modeling population processes. Oxford: Blackwell Publishing.

Riley, J.C. (2001). Rising Life Expectancy. Cambridge University Press. doi:10.1017/cbo9781316036495.

Robine, J.M. (2001). Redefining the stages of the epidemiological transition by a study of the dispersion of life spans: The case of France. Population: An English Selection 13(1): 173-193. http://www.jstor.org/stable/3030265.

Siler, W. (1979). A competing-risk model for animal mortality. Ecology 60(4): 750-757. doi:10.2307/1936612.

Smits, J. and Monden, C. (2009). Length of life inequality around the globe. Social Science \& Medicine 68(6): 1114-1123. doi:10.1016/j.socscimed.2008.12.034.

Vallin, J. and Meslé, F. (2009). The segmented trend line of highest life expectancies. Population and Development Review 35(1): 159-187. doi:10.1111/j.17284457.2009.00264.x.

Vaupel, J.W. (1986). How change in age-specific mortality affects life expectancy. Pop- 
ulation Studies 40(1): 147-157. doi:10.1080/0032472031000141896.

Vaupel, J.W. (2010). Biodemography of human ageing. Nature 464(7288): 536-542. doi:10.1038/nature08984.

Vaupel, J.W. and Gowan, A.E. (1986). Passage to Methuselah: Some demographic consequences of continued progress against mortality. American Journal of Public Health 76(4): 430-433. doi:10.2105/AJPH.76.4.430.

Vaupel, J.W., Zhang, Z., and van Raalte, A.A. (2011). Life expectancy and disparity: an international comparison of life table data. BMJ Open 1(1). doi:10.1136/bmjopen2011-000128.

Vaupel, J. and Canudas-Romo, V. (2003). Decomposing change in life expectancy: A bouquet of formulas in honor of Nathan Keyfitz's 90th birthday. Demography 40(2): 201-216. doi:10.1353/dem.2003.0018.

Wilmoth, J.R. (1997). In search of limits. In: Watcher, K. and Finch, C. (eds.). Between Zeus and the Salmon: the biodemography of longevity. National Academies Press Washington, DC: 38-64.

Wilmoth, J.R. and Robine, J.M. (2003). The world trend in maximum life span. Population and Development Review 29: 239-257. www.jstor.org/stable/3401354.

Wilmoth, J. and Horiuchi, S. (1999). Rectangularization revisited: Variability of age at death within human populations. Demography 36(4): 475-495. doi:10.2307/2648085.

Wrycza, T. (2014). Entropy of the Gompertz-Makeham mortality model. Demographic Research 30(49): 1397-1404. doi:10.4054/DemRes.2014.30.49.

Yashin, A.I., Begun, A.S., Boiko, S.I., Ukraintseva, S.V., and Oeppen, J. (2001). The new trends in survival improvement require a revision of traditional gerontological concepts. Experimental Gerontology 37: 157-167. doi:10.1016/S0531-5565(01)00154-1. 


\section{Appendices}

\section{Appendix A: Changes in variability: effects of $\beta$ and $M$}

We stated that changing the parameter $\beta$ of the Gompertz hazard equation will have an effect on variability of the age at death. In this section, we evaluate this effect by looking at a measure of variability, namely e-dagger $\left(e^{\dagger}\right)$, and attest the contribution of each of the parameters in the Gompertz hazard to the change in variability. Among the different indicators used to measure variability of the age at death (Robine 2001; Wilmoth and Horiuchi 1999; Vaupel, Zhang, and van Raalte 2011), we focus on $e^{\dagger}$, a measure of lifespan disparity often interpreted as the average years of life expectancy lost due to death:

$$
e_{t}^{\dagger}=\int_{0}^{\omega} H_{x, t} l_{x, t} d_{x}
$$

where $l_{x, t}$ is the survival distribution, and $H_{x, t}$ is the cumulative hazard, equal to:

$$
H_{x, t}=e^{\beta_{t}\left(x-M_{t}\right)}-e^{-\beta_{t} M_{t}}=\frac{1}{\beta_{t}} \mu_{x, t}-e^{-\beta_{t} M_{t}} .
$$

Therefore, $e_{t}^{\dagger}$ can be written as

$$
e_{t}^{\dagger}=\frac{1}{\beta_{t}} \int_{0}^{\omega} \mu_{x, t} l_{x, t} d_{x}-e^{-\beta_{t} M_{t}} \int_{0}^{\omega} l_{x, t} d_{x}
$$

leading to

$$
e_{t}^{\dagger}=\frac{1}{\beta_{t}}-e^{-\beta_{t} M_{t}} e_{0, t} .
$$

Wrycza (2014) also showed this relation for Gompertz-Makeham entropy using the standard parametrization. It is possible to quantify the respective effects of $\beta_{t}$ and $M_{t}$ on $e_{t}^{\dagger}$ by looking at its time derivative, denoted by a dot on top of the variable. From equation (A2) changes in $e_{t}^{\dagger}$ over time $\left(\dot{e}_{t}^{\dagger}\right)$ can be expressed by components of changes for both Gompertz parameters:

$$
\dot{e}_{t}^{\dagger}=-\dot{\beta}_{t}\left[\frac{1}{\beta_{t}^{2} e_{0, t}}-M_{t} e^{-\beta_{t} M_{t}}\right] e_{0, t}-\dot{e}_{0, t}\left[e^{-\beta_{t} M_{t}}\right]+\dot{M}_{t}\left[\beta_{t} e^{-\beta_{t} M_{t}} e_{0}\right] .
$$

As shown by equation (6), $\dot{e}_{0, t}$ can be decomposed by a factor of change of $M_{t}$ and $\beta_{t}$. Therefore, $e_{0, t}$ contributions to changes in $e_{t}^{\dagger}$ can be distributed into $M_{t}$ and $\beta_{t}$ contributions, obtaining 


$$
\dot{e}_{t}^{\dagger}=\underbrace{-\dot{\beta}_{t}\left[\frac{1}{\beta_{t}^{2}}-\left(M_{t} e_{0, t}+F_{\beta}\right) e^{-\beta_{t} M_{t}}\right]}_{\delta \beta}+\underbrace{\dot{M}_{t}\left[\left(\beta_{t} e_{0, t}-F_{M}\right) e^{-\beta_{t} M_{t}}\right]}_{\delta M},
$$

where $\delta \beta$ and $\delta M$ are the gains in $e_{t}^{\dagger}$ produced by a change in parameters $\beta_{t}$ and $M_{t}$, respectively, and $F_{\beta}$ and $F_{M}$ are the terms multiplying $\dot{\beta}_{t}$ and $\dot{M}_{t}$ respectively in equation (6):

$$
\begin{gathered}
F_{\beta}=\int_{0}^{\omega} l_{a, t} \int_{0}^{a} \mu_{x, t}\left(\frac{1}{\beta_{t}}+x-M_{t}\right) d_{x} d_{a} \\
F_{M}=\int_{0}^{\omega} l_{a, t} \int_{0}^{a} \beta_{t} \mu_{x, t} d_{x} d_{a} .
\end{gathered}
$$

Table 6 shows an application of the $e_{t}^{\dagger}$ decomposition to Swedish and HMD average female data. It is shown that the main factor of variability changes comes from changes in the parameter $\beta_{t}$. However, increasing the modal age at death produced a small increase in lifespan disparities.

Changes in $e_{t}^{\dagger}$ are thus driven by both Gompertz parameters. In general, increasing $\beta_{t}$ will lead to a smaller variability of the age at death, while increasing $M_{t}$ would have the opposite effect. These results are consistent with the results of Engelman, Caswell, and Agree (2014). Using a Siler model, the authors show that a decrease in the timing parameter for senescent mortality $\left(\alpha_{2}\right)$ will increase the variability (Engelman, Caswell, and Agree 2014). However, Table 6 and Figure 6 show that this variability expansion resulting from a shift in $M_{t}$ will generally be too small to drive substantial changes in lifespan disparities. The assumption that mortality compression is produced by an increase in $\beta_{t}$ is then confirmed. 
Table 6: e-dagger $\left(e_{t}^{\dagger}\right)$ and its decomposition due to changes in the Gompertz parameters, Swedish and HMD countries average, females, 1900, 1950 and 2000

\begin{tabular}{rcccc}
\hline & \multicolumn{3}{c}{ Sweden } & HMD Average \\
& 1900 & 1950 & 2000 & 2000 \\
\hline$e_{t}^{\dagger}$ & 13.0425 & 9.7848 & 8.8384 & 9.2218 \\
$e_{t+5}^{\dagger}$ & 13.4824 & 9.6259 & 8.7529 & 9.0654 \\
$\dot{e}_{t}^{\dagger}$ & 0.4399 & -0.1589 & -0.0855 & -0.1564 \\
& & & & \\
$\delta \beta$ & 0.4356 & -0.1601 & -0.0856 & -0.1567 \\
$\delta M$ & 0.0043 & 0.0012 & 0.0001 & 0.0003 \\
$\delta \beta+\delta M$ & 0.4398 & -0.1589 & -0.0855 & -0.1564 \\
\hline
\end{tabular}

Source: HMD (2015) and authors' own calculation.

Note: By rounding the numbers to the fourth decimal point in the table, the sum of the contributions $\left(\sum \delta_{i}\right)$ might differ slightly from the difference in e-dagger $\left(\dot{e}_{t}^{\dagger}\right)$.

Figure 6: $\quad$ Trends over time of the Gompertz parameters' contribution to changes in e-dagger $\left(e_{t}^{\dagger}\right)$ for females, Sweden and HMD average, 1900-2010

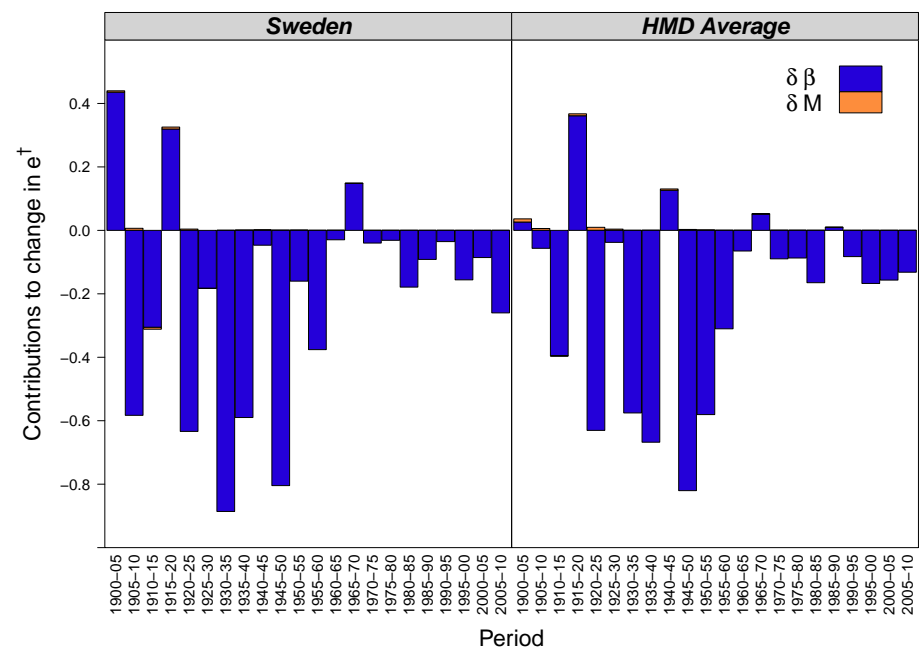

Source: HMD (2015) and authors' own calculation. 


\section{Appendix B: Applying the decomposition to discrete data}

The estimation procedure of our methodology can be done to discrete data by estimating the functions at their midpoint over a certain time interval (Preston, Heuveline, and Guillot 2001; Vaupel and Canudas-Romo 2003). As suggested by Vaupel and Canudas-Romo (2003), if data are available between time $t$ and $t+h$, the midpoint value of the function $v_{x, t}$ was estimated by

$$
v_{x, t+h / 2}=v_{x, t}\left(\frac{v_{x, t+h}}{v_{x, t}}\right)^{1 / 2}
$$

The derivative of the function $v_{x, t+h / 2}$ was estimated by

$$
\dot{v}_{x, t+h / 2}=v_{x, t+h / 2} \frac{\ln \left[\frac{v_{x, t+h}}{v_{x, t}}\right]}{h} .
$$

In some cases, it could make more sense to assume a linear change in the interval (Vaupel and Canudas-Romo 2003). In these cases, we used

$$
v_{x, t+h / 2}=\frac{v_{x, t+h}+v_{x, t}}{2}
$$

and

$$
\dot{v}_{x, t+h / 2}=\frac{v_{x, t+h}-v_{x, t}}{h} .
$$

We used these latter estimates for the change over time of the life expectancy $\left(\dot{e}_{0, t}\right)$. The other functions were estimated by assuming an exponential change, as presented in equations (B1) and (B2). It is important to note that these procedures generate annual estimates, and also that the midpoint of each term multiplying $\dot{\beta}_{t}$ and $\dot{M}_{t}$ in equation (6) should be estimated. For example, the annualized $\Delta M$ for the period $t$ to $t+h$ $\left(\Delta M_{t+h / 2}\right)$ using the Gompertz model is calculated as

$$
\Delta M_{t+h / 2}=\dot{M}_{t+h / 2} \int_{0}^{\omega} l_{x, t+h / 2} \beta_{t+h / 2} H_{x, t+h / 2} d x,
$$

where $H_{x, t}$ is the cumulative hazard at time $t$ and age $x$. In the illustration section, the results are presented for five-year periods. As the above equations are valid for annual changes, the decomposition is applied to yearly differences, which are then summed up to equal for longer periods. Yearly estimates are generally more accurate than the estimates for longer periods. Similar methodology is also applied for the Gompertz-Makeham and Siler models. 


\section{Appendix C: International comparison}

Figure 7: $\quad$ Trends over time of the Gompertz parameters' contribution to changes in female life expectancy at age $30\left(\dot{e}_{30, t}\right)$, HMD countries, 1900-2010

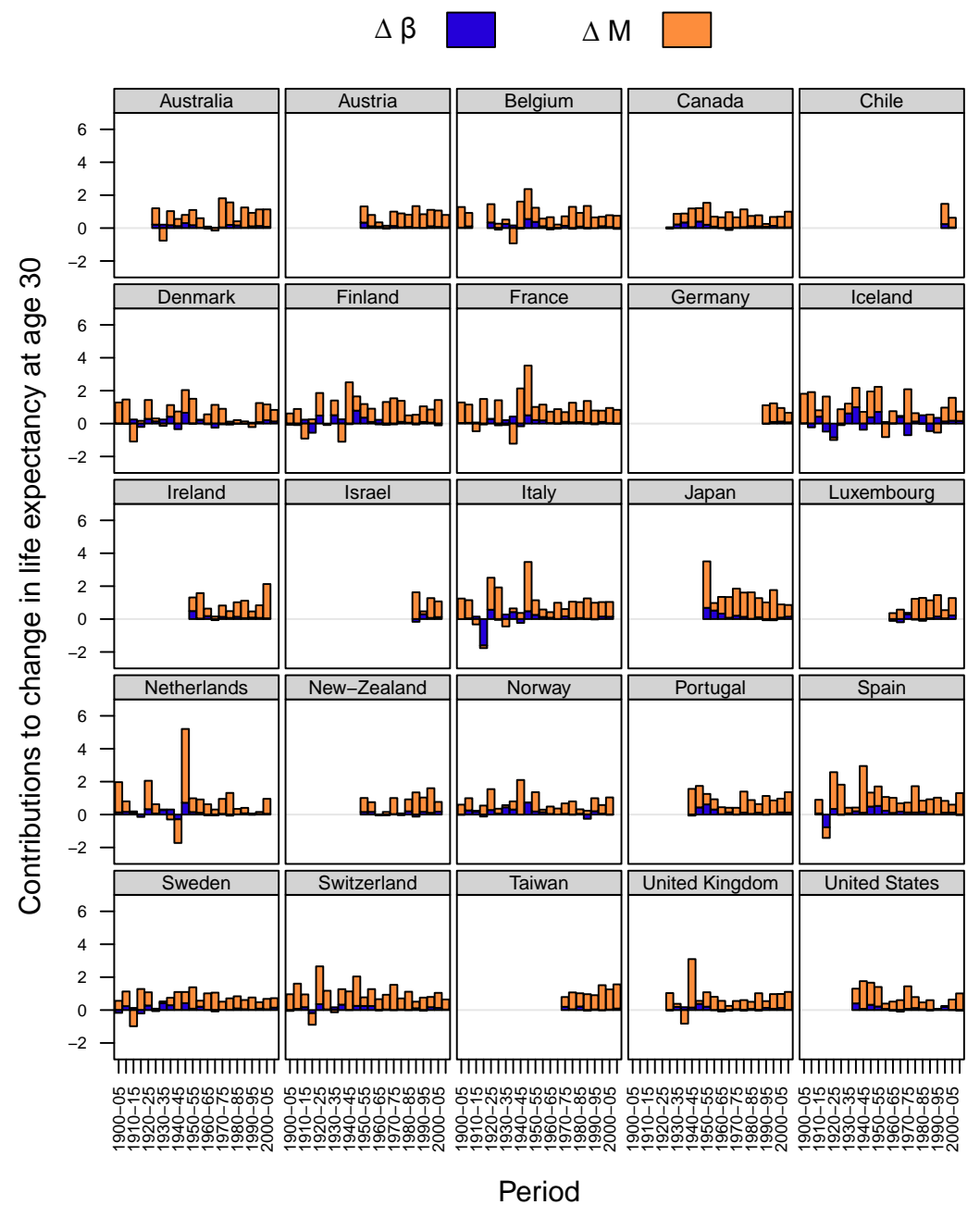

Source: HMD (2015) and author's own calculation. 
Figure 8:

Trends over time of the Gompertz-Makeham parameters' contribution to changes in female life expectancy at age $30\left(\dot{e}_{30, t}\right)$, HMD countries, 1900-2010.

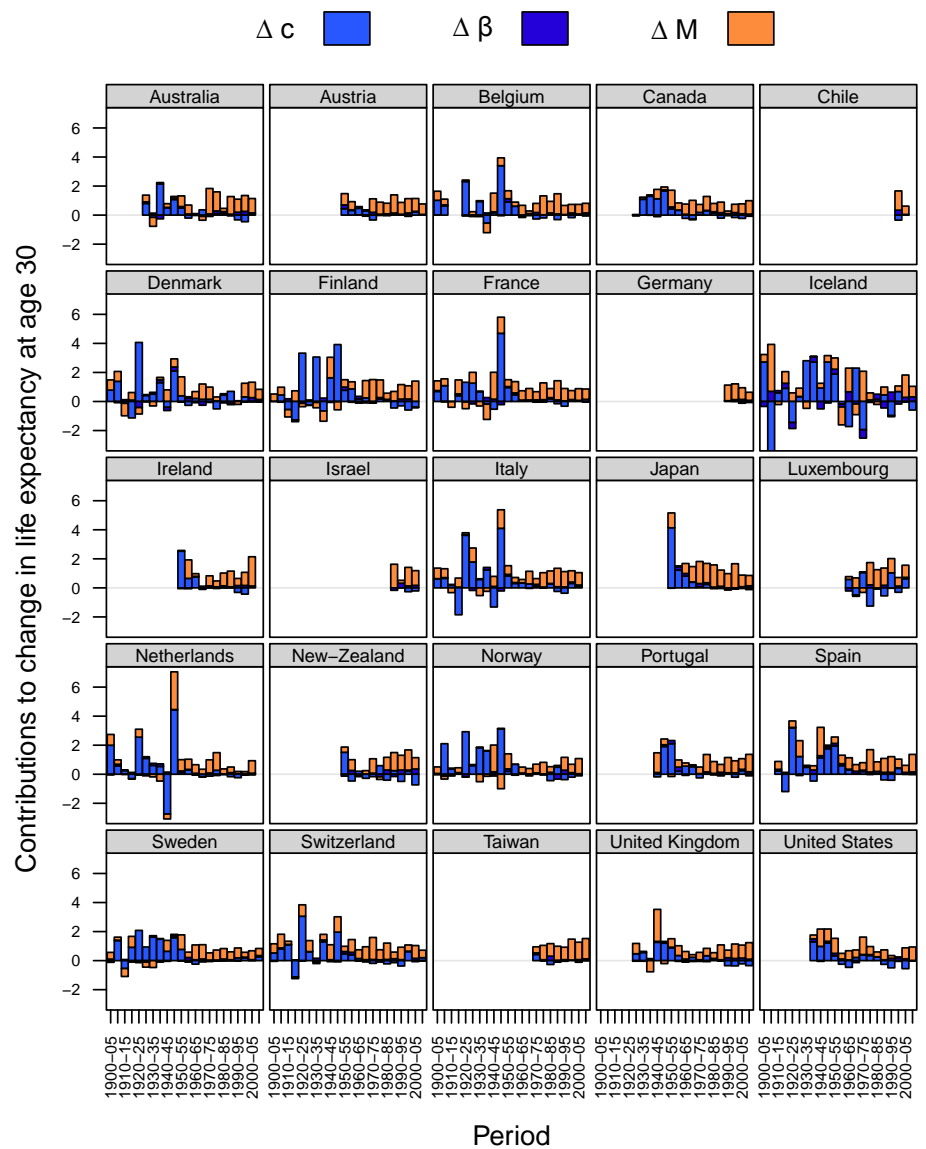

Source: HMD (2015) and authors' own calculation. 
Figure 9: $\quad$ Trends over time of the Siler parameters' contribution to changes in female life expectancy at age $0\left(\dot{e}_{0, t}\right)$, HMD countries, 1900-2010

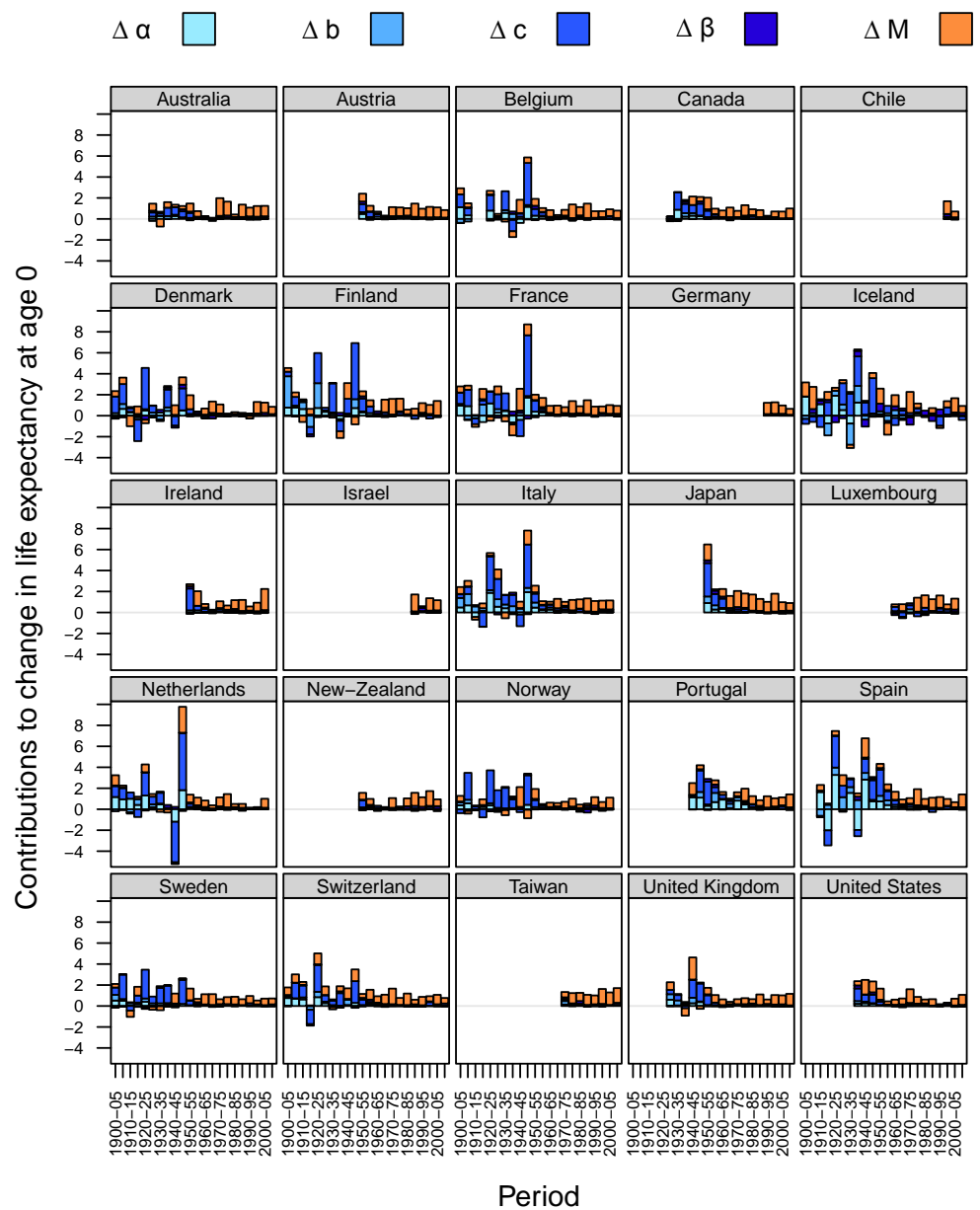

Source: HMD (2015) and authors' own calculation. 


\section{Appendix D: Relative differences}

Figure 10: $\quad$ Female life expectancy at birth $\left(e_{0, t}\right)$ and relative gain in life expectancy due to changes in the Siler parameters, Sweden and HMD average, 1900-2010. The black and red lines are the life expectancy at birth observed (in black) and modeled (in red) presented in Figure 5.

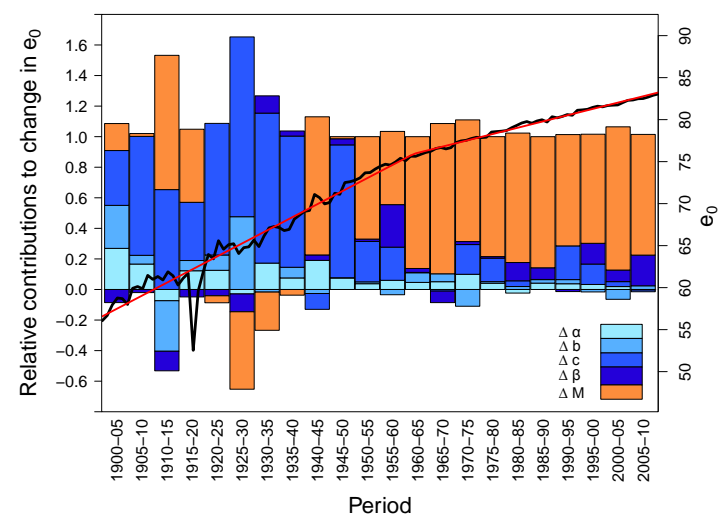

a) Sweden

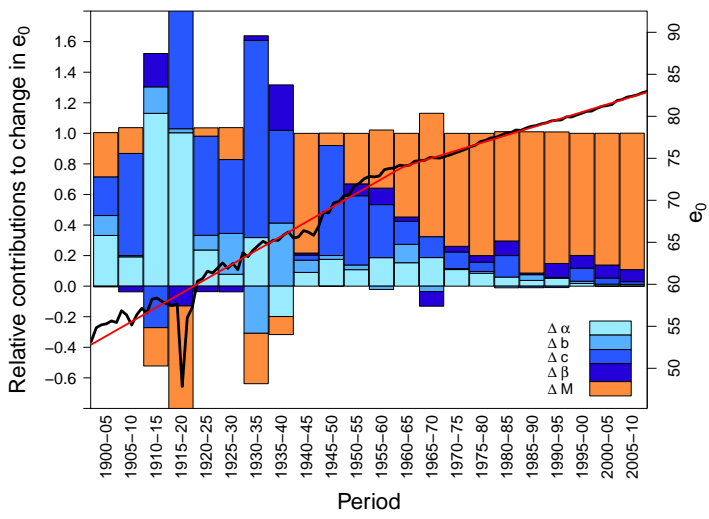

b) HMD average

Source: HMD (2015) and authors' own calculation.

Note: The sum of the contributions $\left(\sum \Delta_{i}\right)$ for each bar equal to 1 in this figure. 


\section{Appendix E: Life expectancy decomposition: Gompertz, Logistic and Weibull models}

Table 7: $\quad$ Female life expectancy at age $30\left(e_{30, t}\right)$ and its decomposition due to changes in the modal age at death $(\Delta M)$ and shape $(\Delta \beta)$, using Gompertz, Logistic and Weibull models, Sweden and HMD average, 1900, 1950 and 2000

\begin{tabular}{|c|c|c|c|c|c|}
\hline & \multicolumn{3}{|c|}{ Sweden } & \multicolumn{2}{|c|}{ HMD Average } \\
\hline & 1900 & 1950 & 2000 & 2000 & $(\min , \max )$ \\
\hline \multicolumn{6}{|c|}{ Gompertz } \\
\hline$e_{30, t}$ & 37.82 & 44.18 & 52.04 & 51.43 & $(49.23,54.79)$ \\
\hline$e_{30, t+5}$ & 38.21 & 45.56 & 52.72 & 52.45 & $(50.40,55.67)$ \\
\hline$\dot{e}_{30, t}$ & 0.39 & 1.39 & 0.68 & 1.03 & \\
\hline$\Delta \beta$ & -0.17 & 0.09 & 0.05 & 0.09 & $(-0.11,0.22)$ \\
\hline$\Delta M$ & 0.56 & 1.30 & 0.63 & 0.94 & $(0.48,2.08)$ \\
\hline$\Delta \beta+\Delta M$ & 0.39 & 1.39 & 0.68 & 1.03 & \\
\hline \multicolumn{6}{|c|}{ Logistic } \\
\hline$e_{30, t}$ & 37.93 & 44.25 & 52.13 & 51.50 & $(49.30,54.90)$ \\
\hline$e_{30, t+5}$ & 38.33 & 45.64 & 52.80 & 52.53 & $(50.46,55.78)$ \\
\hline$\dot{e}_{30, t}$ & 0.40 & 1.39 & 0.67 & 1.02 & \\
\hline$\Delta \beta$ & -0.17 & 0.07 & 0.04 & 0.08 & $(-0.15,0.22)$ \\
\hline$\Delta M$ & 0.57 & 1.32 & 0.62 & 0.94 & $(0.48,2.12)$ \\
\hline$\Delta \beta+\Delta M$ & 0.40 & 1.39 & 0.67 & 1.02 & \\
\hline \multicolumn{6}{|c|}{ Weibull } \\
\hline$e_{30, t}$ & 38.50 & 44.33 & 52.09 & 51.46 & $(49.36,55.05)$ \\
\hline$e_{30, t+5}$ & 38.98 & 45.77 & 52.74 & 52.47 & $(50.47,55.88)$ \\
\hline$\dot{e}_{30, t}$ & 0.48 & 1.44 & 0.65 & 1.01 & \\
\hline$\Delta \beta$ & 0.05 & 0.02 & 0.02 & 0.04 & $(-0.03,0.08)$ \\
\hline$\Delta M$ & 0.42 & 1.41 & 0.62 & 0.97 & $(0.50,2.10)$ \\
\hline$\Delta \beta+\Delta M$ & 0.48 & 1.44 & 0.65 & 1.01 & \\
\hline
\end{tabular}

Source: HMD (2015) and authors' own calculation.

Note: By rounding the numbers to the second decimal point in the table, the sum of the contributions $\left(\sum \Delta_{i}\right)$ might differ slightly from the difference in life expectancy $\left(\dot{e}_{30, t}\right)$. 
Bergeron-Boucher, Ebeling \& Canudas-Romo: Decomposing changes in life expectancy 